\title{
Reciprocating Feed System Development Status
}

\author{
James B. Blackmon ${ }^{1}$ and David E. Eddleman ${ }^{2}$ \\ Propulsion Research Center, University of Alabama in Huntsville, Huntsville Al,35899
}

\begin{abstract}
I. Abstract
The reciprocating feed system (RFS) is an alternative means of providing high pressure propellant flow 1 at low cost and system mass, with high fail-operational reliability. The RFS functions by storing the liquid propellants in large, low-pressure tanks and then expelling each propellant through two or three small, high-pressure tanks. Each RFS tank is sequentially filled, pressurized, expelled, vented, and refilled so as to provide a constant, or variable, mass flow rate to the engine. This type of system is much lighter than a conventional pressure fed system in part due to the greatly reduced amount of inert tank weight. The delivered payload for an RFS is superior to that of conventional pressure fed systems for conditions of high total impulse and it is competitive with turbopump systems, up to approximately 2000 psi. An advanced version of the RFS uses autogenous pressurization and thrust augmentation to achieve higher performance. In this version, the pressurization gases are combusted in a small engine, thus making the pressurization system, in effect, part of the propulsion system. The RFS appears to be much less expensive than a turbopump system, due to reduced research and development cost and hardware cost, since it is basically composed of small high pressure tanks, a pressurization system, and control valves. A major benefit is the high reliability fail-operational mode; in the event of a failure in one of the three tank-systems, it can operate on the two remaining tanks. Other benefits include variable pressure and flow rates, ease of engine restart in micro-gravity, and enhanced propellant acquisition and control under adverse acceleration conditions. We present a system mass analysis tool that accepts user inputs for various design and mission parameters and calculates such output values payload and vehicle weights for the conventional pressure fed system, the RFS, the Autogenous Pressurization Thrust Augmentation (APTA) RFS, and turbopump systems. Using this tool, a preliminary design of a representative crew exploration vehicle (CEV) has been considered. The design parameters selected for a representative system were modeled after the orbital maneuvering system (OMS) on the Shuttle Orbiter, with an increase of roughly a factor of ten in the delta$\mathrm{V}$ capability and a greater thrust $(30,000 \mathrm{lbs}$, vs. $12,000 \mathrm{lbs})$. Both storable and cryogenic propellants were considered. Results show that a RFS is a low mass alternative to conventional pressure fed systems, with a substantial increase in payload capability and that it is weight-competitive with turbopump systems at low engine pressure (a few hundred psi); at high engine pressures, the APTA RFS appears to offer the highest payload. We also present the status of the RFS test bed fabrication, assembly, and checkout. This test bed is designed to provide flow rates appropriate for engines in the roughly 10,000 to $30,000 \mathrm{lb}$ thrust range.
\end{abstract}

\section{Technical Discussion}

The basic concept for a RFS is covered in patent number 6,314,978 B1 titled Reciprocating Feed System for Fluids ${ }^{1}$ and in an early AIAA Joint Propulsion Conference paper ${ }^{2}$. This patent was originally assigned to McDonnell Douglas, but was donated to the University of Alabama in Huntsville in 2005. Other UAH patents related to the RFS are pending. Other work related to this topic is addressed by Knight $^{3}$, by Flowmetrics ${ }^{4}$, and in patent number 3,213,804, titled Fluid Pressurizing System ${ }^{5}$. The RFS contains propellant in both of the main, low pressure, storage tanks and in two or three small, high pressure tanks. The small tanks would be filled initially before engine firing and would also fill after engine shutdown for multiple restart missions. The small tanks would alternately expel fluid into the engine, vent, refill with fluid from the main tank, and then be pressurized. This controlled, alternating cycle provides a uniform, pressurized flow of fuel and oxidizer to the engine. The use of small, lightweight tanks allows the RFS to operate at engine pressure ranges above those normally associated with pressure fed systems.

\footnotetext{
${ }^{1}$ Research Professor, Department of Mechanical and Aerospace Engineering, University of Alabama in Huntsville, AIAA Associate Fellow

${ }^{2}$ Graduate Student, Department of Mechanical and Aerospace Engineering, University of Alabama in Huntsville, AIAA Member.
} 
The RFS offers benefits in terms of weight and cost savings, performance, and reliability. The weight savings in comparison to conventional pressure fed system are primarily in the greatly reduced main tank weights, and are valid in general for relatively large amounts of propellant, as with high total impulse systems. Although the RFS requires several more valves for fluid and pressurization control, some additional lines, and two or three small, high pressure tanks, our mass estimates show that these weights are less than the weight savings associated with the low pressure main storage tank. More importantly, operation at higher chamber pressures for a constrained nozzle exit diameter, results in smaller engines with higher expansion ratios and greater specific impulse, with a significant overall improvement in delivered payload. Also, the RFS system weight is comparable to that of a turbopump system, up to pressures of the order of 1500 to 2000 psi. A new approach for pressurizing the RFS tanks, termed the Autogenous Pressurization Thrust Augmentation (APTA) RFS (patent pending), appears to offer greater payload capability than turbopump systems. One way to represent the region of application for the RFS is shown in Figure 1, where the central region of the Pressure vs. Total Impulse curve is occupied by the RFS. The region of application of the RFS is shown in central region, with some overlap with turbopump systems for several hundred to about 1500 psi chamber pressures.

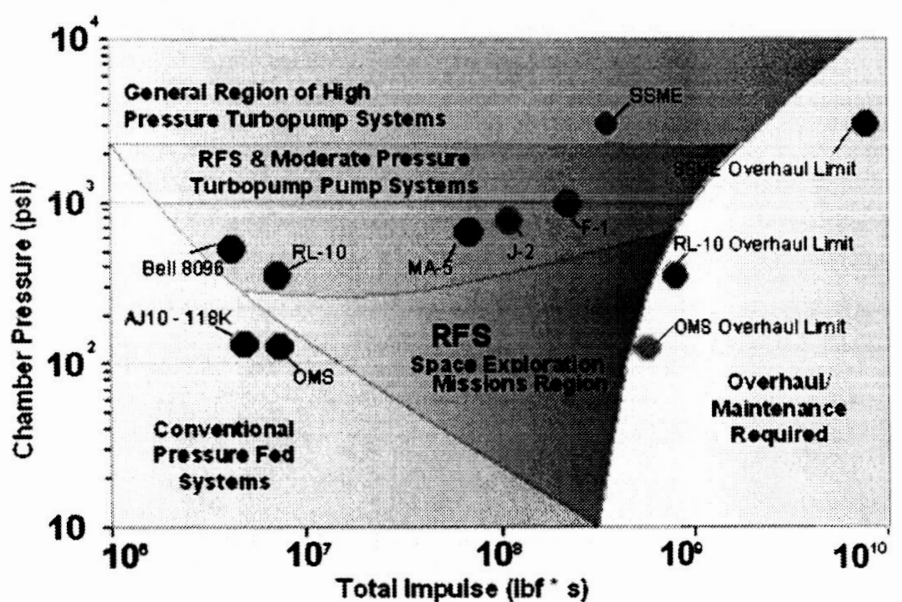

Figure 1 - Representative Regions of Application for Pump Fed, RFS, and Turbopump Systems
The cost of the RFS is expected to be substantially lower than the cost of a turbopump system. Some of this difference in cost is due to the relatively high research and development costs associated with turbopumps, compared to the relatively low costs associated with the RFS flow control; hardware costs for the additional valves, small tanks, and pressurization gas system should be much less than for the turbopump system.

There are a number of other features that further reduce weight and cost. The RFS allows use of a smaller, higher chamber pressure and higher performance engine;

engine performance is improved through the higher expansion ratio achieved with a smaller, higherpressure engine, for a given vehicle envelope. The smaller combustion chamber also reduces the overall mass and volume of the spacecraft. However, for the RFS, it is necessary to compare the increased engine pressure and performance with the increased pressurization gas and high-pressure bottle weights required; we show that overall, there is an increase in delivered payload for representative systems and propellant combinations. Propellant acquisition and control in micro-gravity in the smaller RFS tanks would be easier than from a large tank, especially when it is nearly empty. This condition offers greater assurance of engine restart in micro-gravity, without gas ingestion, which could lead to propulsion instabilities and damage. Use of smaller tanks also offers greater assurance of propellant delivery under adverse acceleration conditions. The RFS would also not require the spin up time associated with that a turbopump, especially for cryogenic propellants, and we anticipate that in missions involving multiple restarts, it would offer a substantial operational advantage. The RFS would also allow for a relatively fast variation of fuel or oxidizer engine inlet pressure. This could be achieved simply by having one of the RFS tanks pressurized to a different level during its cycle. The other RFS tanks would each then do the same during their respective pressurization periods. Control valves could also be used to rapidly vary the flow rate. This capability would allow for deep throttling of engines, which may be useful for ascent/descent, docking, maneuvering, etc.

The basic principle of the RFS allows just two small tanks to be used for propellant feed to the engine. However, by using three tanks the RFS offers at least a partial fail-operational mode in the event of a hardware failure in one of the three tank systems; with little additional weight, four tanks can be used, for additional redundancy. We anticipate that the resulting overall system weight and cost savings, improved reliability, and improved performance will allow missions of greater payload and enhanced capability to be 
flown than with conventional pressure-fed systems, and it would further support the use of cryogenic propellants for advanced engines (i.e., liquid hydrogen or methane with liquid oxygen), since it would not require development of a new turbopump system.

The size of the high-pressure RFS tanks is in part determined by the total system size and by the flow rate. Given that there is a certain response time required to refill each small tank and then re-pressurize it, sizing the tank would be in part a function of the flow rate, especially since the high flow rates cause turbulent sloshing, bubble formation, and these fluid dynamic effects must allowed to subside. Therefore, low flow rate systems could use smaller RFS tanks than high flow rate systems. Similarly, the flow rate and the vehicle acceleration level have to be considered in refill times. Typically, we assume that each RFS tank can be refilled in seconds, and this can provide sufficient time to vent, refill, and pressurize each tank such that it is ready to expel liquid when required as part of the reciprocating cycle. However, we've found that the refill time has a secondary effect on the system mass, and therefore longer times can be used as needed.

We developed and have been refining a system mass analysis tool in Excel that compares the RFS with several versions of conventional pressure fed and turbopump systems, with various propellant combinations. The feed system and consumable mass estimates include main tanks, RFS tanks, valves, lines, pressurization gas bottles, tank insulation, propellant, and pressurization gas, support structure, etc. The program provides for various configurations of the main tanks, RFS tanks, and pressurization gas bottles with options for storage of the smaller RFS and pressurization bottles internal or external to the main tanks. We also have various factors for miscellaneous hardware, a vehicle diameter restriction, various tables of values for propellant and pressurization gas properties, material properties, etc. The analyses are based on inputs of such variables as thrust, burn duration, mixture ratio, specific impulse, safety factors, storage and operating temperatures, ullage gas volumes and temperatures, residual volumes, and tank and line material properties. Tables of physical properties for propellants and pressurization gases are used to obtain density as function of temperature and pressure. Conventional values are used as default values for various parameters, but these values can be easily changed by the user. A number of the mass estimating relationships are used from standard aerospace references ${ }^{6}$, in addition to fundamental analyses or vendor-supplied data. Results are then calculated and plotted automatically for viewing and also shown in tabular form for more precise results. We developed this initial analysis using the major component masses such as tank, propellant and pressurization masses, etc. We have also added a new variation of the RFS, in which the inert gas pressurization system is replaced with an autogenous pressurization system; the pressurization gas for the fuel tank is a fuel (e.g., gaseous hydrogen) with gaseous oxygen used for the $\mathrm{LO}_{2}$ tank. The RFS high-pressure gases are vented sequentially through an auxiliary engine. This Autogenous Pressurization Thrust Augmentation (APTA) RFS in effect turns the pressurization system into a high performance enhancement to the propulsion system, and substantially increases the payload capability.

For comparison purposes, we have assumed a Crew Exploration Vehicle (CEV) system modeled after the Space Shuttle Orbiter Orbital Maneuvering System (OMS), to which we have made several modifications to account for the type of mission a CEV might be used for. The OMS system is a conventional pressure fed monomethylhydrazine/nitrogen tetraoxide $\left(\mathrm{MMH} / \mathrm{N}_{2} \mathrm{O}_{4}\right)$ system that operates at a tank pressure of approximately 250 psi and an engine pressure of approximately $125 \mathrm{psi}$. It provides roughly $1000 \mathrm{ft} / \mathrm{sec}$ of delta-V with the two 6,000 lbf thrust engines, with an Orbiter weight of approximately $180,000 \mathrm{lbs}$, and a payload of approximately $40,000 \mathrm{lbs}$. For our example, we assumed a burn time of the order of 1000 seconds, with a delta $\mathrm{V}$ of $4,000 \mathrm{ft} / \mathrm{s}$, with a choice of three propellants: $\mathrm{MMH} / \mathrm{N}_{2} \mathrm{O}_{4}, \mathrm{LH}_{2} / \mathrm{LO}_{2}$, and $\mathrm{LO}_{2} / \mathrm{RP}-1$. We also assumed a structural mass estimate of $150,000 \mathrm{lbs}$ for the vehicle. Payload weights were then determined for the various options. We used a higher delta-V on the assumption that a CEV would require a substantial maneuvering capability, higher payload capability, space-based maintenance, and growth potential. Realistic values, traceable to various space systems, were used for other parameters. For example, we have incorporated the vacuum specific impulse as a function of

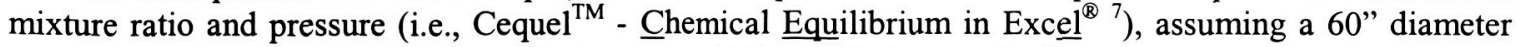
envelope restriction on the nozzle exit diameter. This restriction results in a variation of expansion ratio, mixture ratio, and vacuum specific impulse, with pressure (Table 1). Using these results in our sizing code allows the system to be sized for the optimum engine, with specific mixture ratios and specific impulse inserted for each case. By adjusting the burn times to have the same total propellant weights in all cases, we have a basis for a common comparison. 


\begin{tabular}{|c|c|c|c|c|c|c|c|c|c|c|c|c|c|}
\hline \multicolumn{4}{|c|}{ LOXRP-1, Equilibrium Flow } & \multicolumn{5}{|c|}{ LOXHH2 (L), Equilibrium Flow } & \multicolumn{5}{|c|}{ MMH (L)N2O4 (L), Equilibrium Flow } \\
\hline Exit Diameter (in) & 60 & 60 & 60 & Exit Diameter (in) & 60 & 60 & 60 & 60 & Exit Diameter (in) & 60 & 60 & 60 & 60 \\
\hline Pc (Psi) & 100 & 500 & 1000 & $\mathrm{PC}(\mathrm{Psi})$ & 2000 & 100 & 500 & 1000 & $\mathrm{Pc}(\mathrm{Psi})$ & 100 & 100 & 500 & 1000 \\
\hline Thrust (lbf) & 30000 & 30000 & 30000 & Thrust (bf) & 30000 & 30000 & 30000 & 30000 & Thrust (lbf) & 30000 & 30000 & 30000 & 30000 \\
\hline OF Ratio & 2.7 & 3 & 3.1 & OF Ratio Guess & 5.94 & 4.09 & 5.06 & 5.5 & OF Ratio Guess & 2.1 & 2.1 & \begin{tabular}{|l|}
2.41 \\
\end{tabular} & 2.47 \\
\hline Thrust Coefficient & 1.734 & 1.980 & 2.058 & Thrust Coefficient & 2.007 & 1.684 & 1.883 & 1.949 & Thrust Coefficient & 1.725 & \begin{tabular}{|l|l|}
1.725 \\
\end{tabular} & 1.951 & 2.013 \\
\hline ISP Vac & 319.8 & 357.0 & 369.5 & IsP Vac & 484.9 & 435.4 & 467.3 & 476.9 & IsP Vac & 321.8 & 321.8 & 352.6 & 361.9 \\
\hline Throat Area & 173.04 & 30.31 & 14.58 & Throat Area & 7.47 & 178.14 & 31.86 & 15.39 & Throat Area & 173.93 & 173.93 & 30.76 & 14.90 \\
\hline Area Ratio & 16.3 & \begin{tabular}{|l|}
93.3 \\
\end{tabular} & 193.9 & Area Ratio & 378.4 & 15.9 & 88.8 & 183.7 & Area Ratio 2 & 16.3 & 16.3 & 91.9 & 189.7 \\
\hline Throat Diameter & 14.843 & \begin{tabular}{|l|l|}
6.212 \\
\end{tabular} & 4.308 & Throat Diameter & 3.085 & 15.061 & 6.369 & 4.427 & Throat Diameter & 14.881 & 14.881 & 6.258 & 4.356 \\
\hline
\end{tabular}

Table 1-Equilibrium Thermochemical Analysis Results - 30,000 lb Thrust Engine, Vacuum Specific Impulse for Specified Exit Nozzle Diameter Constraint

We also include tank configuration, tank and line material properties, storage and operating temperatures, residual volumes, unused pressurization gas (including thermodynamic blow down assumptions), etc. We have made all of the design parameters equal for comparisons within the propellant class selected, and the propellant feed system options, so that the comparative results can be considered as representative. The objective here was to have a representative in-space system to show the variation in payload that occurs, for all basic parameters held constant (total propellant mass, delta $\mathrm{V}$, thrust, vehicle dry weight (less the propulsion system and propellant, pressurization system, etc.). The main output is the payload capability for each option, as a function of chamber pressure. Figure 2 shows a representative input/output sheet for the code.

Figures 3 to 5 show the payload vs chamber pressure data for the $\mathrm{LO}_{2} / \mathrm{RP}-1, \mathrm{~N}_{2} \mathrm{O}_{4} / \mathrm{MMH}$, and $\mathrm{LO}_{2} / \mathrm{LH}_{2}$ systems. These results show that an RFS can offer comparable payload masses relative to a turbopump at low and mid-range tank pressures (100 to $1000 \mathrm{psi}$ ). In particular, the Autogenous Pressurization Thrust Augmentation (APTA) RFS offers a substantial increase in payload, for the $\mathrm{LH}_{2} / \mathrm{LO}_{2}$ system. This performance improvement is the result of combusting the pressurization gases, $\mathrm{G}_{2} / \mathrm{GH}_{2}$, in an auxiliary thruster, to increase the total thrust. The analysis also shows that a cryogenic propulsion system for this type of in-space vehicle could be beneficial. As can be seen, the RFS offers an alternative to conventional turbopump systems in terms of possible payload, and greatly extends the payload compared to a conventional pressure fed system, while offering potential safety, reliability, and cost advantages.

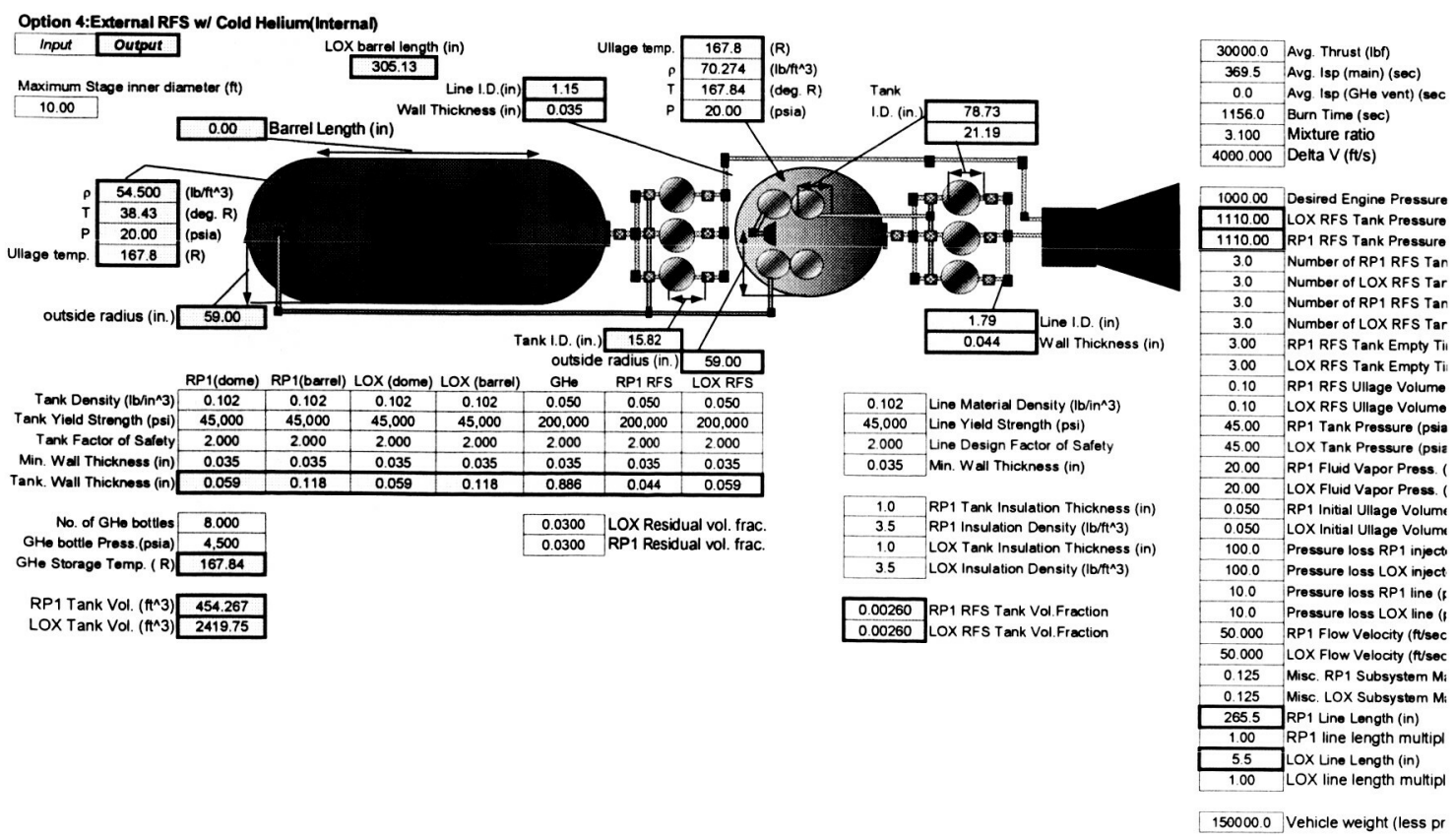

Figure 2 - Representative Input/Output Sheet for PRC Sizing Code 


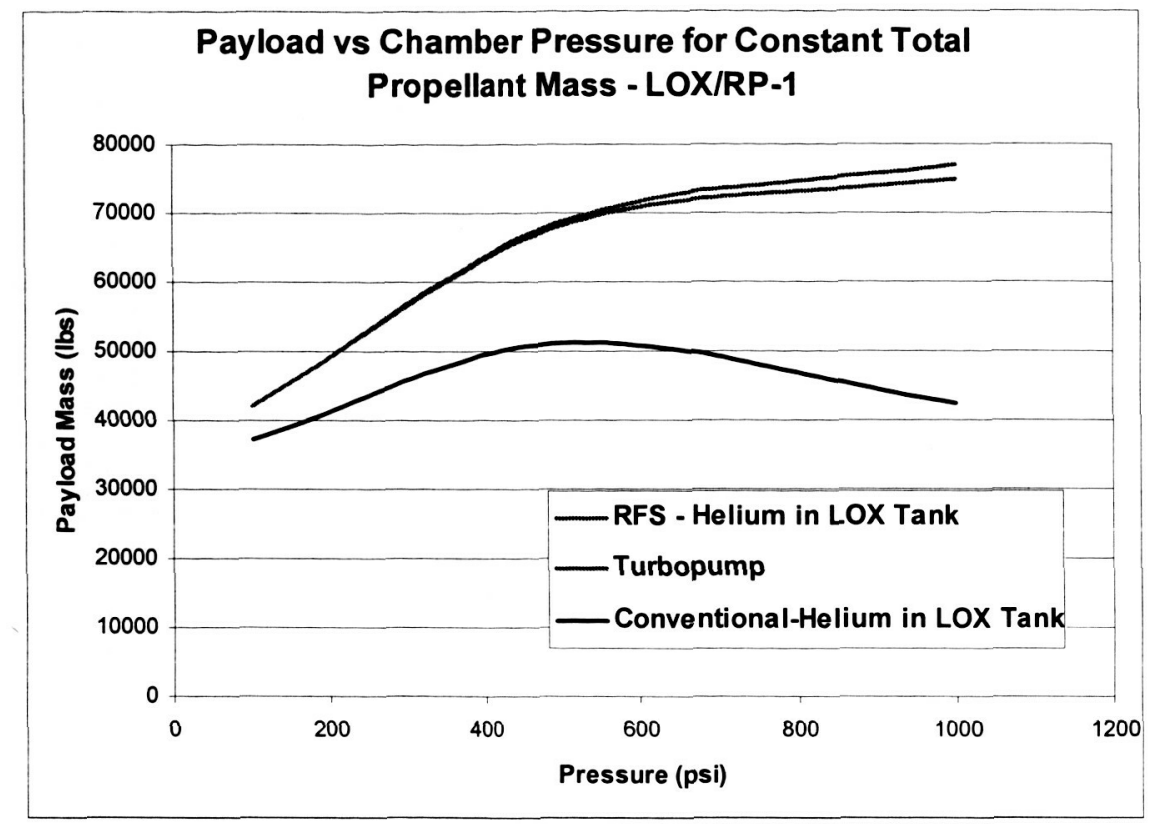

Figure 3: Analysis Tool Comparison of the Payload Massas a Function of Tank Pressure for a LOX/RP-1 System

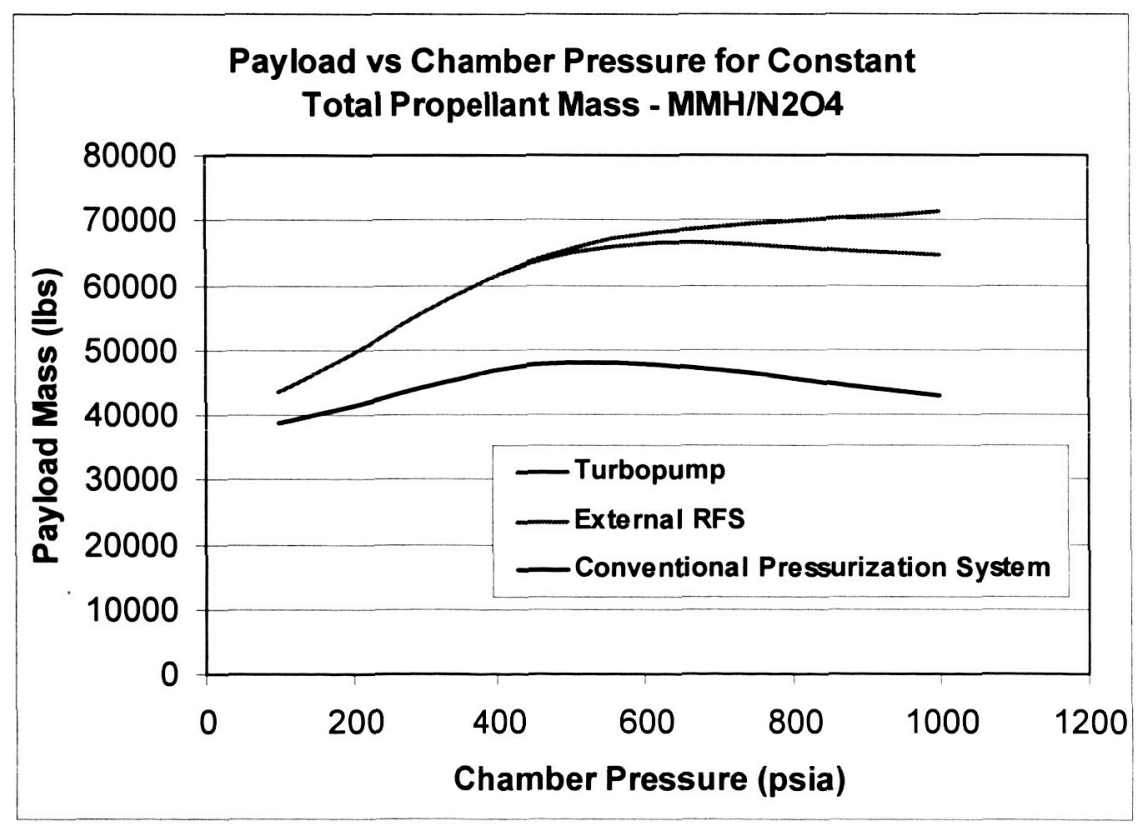

Figure 4 - Analysis Tool Comparison of the Payload Mass as a Function of Tank Pressure for an $M M H / N_{2} \mathrm{O}_{4}$ System 


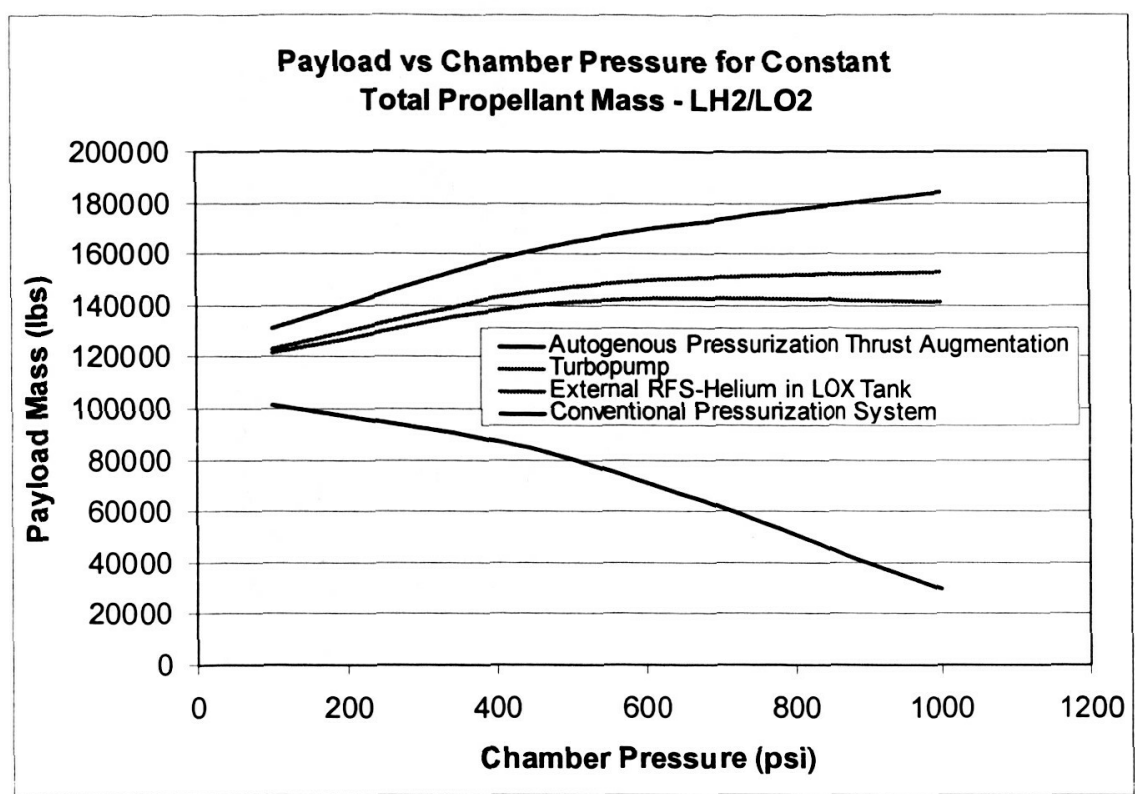

Figure 5 - Analysis Tool Comparison of the Payload Mass as a Function of Tank Pressure for an $\mathrm{LO}_{2} / \mathrm{LH}_{2}$ System

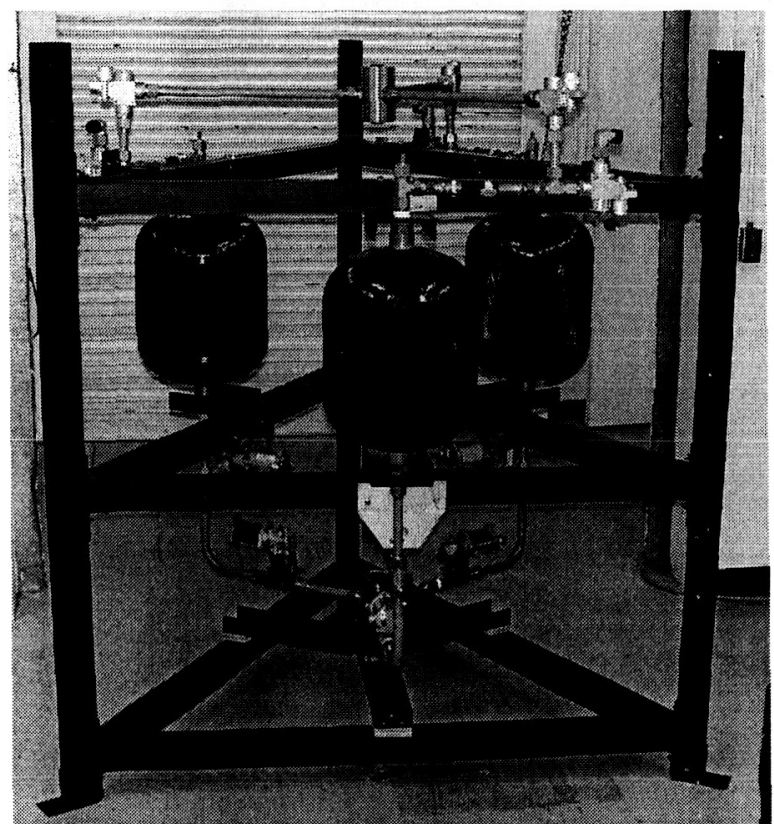

Figure 6-Reciprocating Feed System Test Bed

\section{RFS Test Bed}

UAH is currently developing, with NASA MSFC, a representative RFS system. The RFS test bed is shown in Figure 6. De-ionized water will be used for the initial tests, supplied from a low pressure, 10,000-gallon tank to simulate a storable propellant. The pressurization gas will be missile grade air supplied by the NASA MSFC cross-country line. The test article has three 10-gallon composite over-wrapped pressure vessels rated at 2800 psi for the RFS run tanks. One-inch, pneumatically actuated ball valves will be used for the fill and drain cycles for each tank. Oneinch solenoid valves will be used for venting and pressurization. Initial testing will be conducted at approximately 500 psig. Since tank level sensors are not available at this time, the fill cycle will be calibrated by filling the tanks for prescribed periods of time and capturing the water and recording the volume as it is released. A similar process of catching water as it is released will be used to calibrate the timing of the drain cycle. The outflow will be controlled with a valve to ensure sufficient time for the vent, fill, and pressurization sequences. Later versions will include liquid level sensors. The main pressurization gas supply pressure will be reduced to the desired exit fluid outflow pressure. This will allow for the pressurization valves to remain open during the drain cycle of each tank. Inflow will be controlled by initially having the vent valve closed to prevent loss of liquid during the refill.

This system will be capable of flow rates up to approximately $35 \mathrm{lbm} / \mathrm{sec}$, which corresponds to thrust levels of the order of $30,000 \mathrm{lbf}$, for a complete RFS system. The control system for the valves and data acquisition is completely automated, with real time data acquisition and plotting capability. Data acquisition includes pressure measurements of each of the run tanks, the main supply tank, and the water (inflow and outflow), mass flow rates, and temperatures. Thermocouples will be placed in various positions in the system, primarily to record gas temperature variations during vent, pressurization, and expulsion. Once cycle times are calibrated for a given pressure, it will be determined if common uniform outflow can be determined by cycling through the run tanks at periods on the order of 10-20 seconds. We plan to test the system to the limits of the cycle times, to determine the dimensionless parameters (Weber, Froude Numbers, etc.) that are limiting for refill, pressurization, venting, and expulsion. Other, more 
advanced problems such as failure modes and fail-operational recovery, throttling, effects of valve opening and closing rates, and valve timing will also be investigated.

\section{Conclusion}

The RFS offers a new propellant feed system option for advanced spacecraft such as the planned CEV, as well as launch vehicles. Possible improvements in development time and costs, safety, reliability, performance, and payload weight for a vehicle make this an option that should warrant future study. Our analyses indicate that such a system could be a promising choice for engine pressures up to approximately 1000 to 2000 psi. The RFS may be particularly suited for advanced vehicle systems such as the CEV that would require a reliable, fail operational system capable of performing multiple restarts, with deep throttling, and with a requirement for space-based maintentance. Our analyses also show that $\mathrm{LH}_{2} / \mathrm{LO}_{2}$ systems could double the payload for a vehicle such as the CEV, relative to LOX/RP-1 and MMH/ $\mathrm{N}_{2} \mathrm{O}_{4}$, if used with an RFS or turbopump. Further, with the Autogenous Pressurization Thrust Augmentation RFS, payload could be increased an additional $20 \%$.

\section{Acknowledgements}

This work was supported by NASA Grant NCC8-200 with NASA MSFC with Mary Trawek as the COTR for NASA and Dr. Clark W. Hawk as Principal Investigator for the UAH Propulsion Research Center. We wish to thank Dr. Robert Frederick for the Cequel propulsion system performance calculations, and Chris Morton and Sean Entrekin for their assistance in the preparation of this paper. We also wish to thank NASA MSFC for their support of the RFS Test Bed development, and in particular, Mr. Kevin Pedersen, Mr. Tim Ezell, Mr. Peter Rodriguez, Ms. Becky Farr, and other members of their team.

\section{References:}

${ }^{1}$ Lanning, Mark E. and Blackmon, James B. Boeing Company, Huntington Beach, CA, Patent Application for a "Reciprocating Feed system for Fluids", Patent No.6,314,978, granted November $13^{\text {th }}, 2001$.

${ }^{2}$ Eddleman, David E., Blackmon, James B, and Moser, Marlow D., Reciprocating Feed System for In-Space Propulsion Systems. Joint Propulsion Conference, Huntsville, AL, 20-23 July 2003.

${ }^{3}$ Knight, Andrew "The Race for Low -Cost Launch Vehicles-A New Rocket Engine Design", High Power Rocketry, August 2002.

${ }^{4}$ Harrington, Steve, "Pistonless Dual Chamber Rocket Fuel Pump: Testing and Performance," AIAA 2003-4479.

${ }^{5}$ Sobey, Alfred J. Patent Application for "Fluid Pressurizing System", Patent No. 3,213,804, granted Oct. $26^{\text {th }}, 1965$.

${ }^{6}$ Huzel, Dieter K., and Huang, David H., Modern Engineering for Design of Liquid-Propellant Rocket Engines. American Institute of Aeronautics and Astronautics, 1992.

${ }^{7}$ Cequel, Available from Software and Engineering Associates. See www.seainc.com. 